\title{
Ilio-psoas abscess in neonates
}

\author{
K. A.Edgar ${ }^{1}$, A.E. Schlesinger ${ }^{1}$, R.M.Royster ${ }^{2}$, V.F.X.Deeney ${ }^{3}$ \\ ${ }^{1}$ Department of Radiology, University of Michigan Hospitals, C. S. Mott Children's Hospital, 200 E. Hospital Drive, Ann Arbor, \\ MI 48109-0252, USA \\ ${ }^{2}$ Department of Orthopedic Surgery, Wilford Hall U.S. A. F. Medical Center, Lackland, A. F. B., Texas 78236-5300, USA \\ ${ }^{3}$ Department of Orthopedic Surgery, The Oschner Clinic, 1514 Jefferson Highway, New Orleans, LA 70121, USA
}

Received: 18 June 1992/Accepted: 17 August 1992

\begin{abstract}
We report two cases of primary ilio-psoas abscess in neonates diagnosed by CT and sonography. Iliopsoas abscess is extremely uncommon in this age group.
\end{abstract}

Ilio-psoas abscess is uncommon in children, and very rare in the neonate. To our knowledge, only two previous cases of ilio-psoas abscess in neonates have been reported $[1,2]$. We present two additional cases of ilio-psoas abscess presenting in the neonatal period. CT scans identified the abscess in both patients and provided guidance for needle aspiration in one case. Sonography demonstrated the abscess in the one case in which it was performed.

\section{Case reports}

\section{Case I}

This white male was born at thirty-six weeks gestation by induced vaginal delivery for $\mathrm{Rh}$ incompatibility. An umbilical venous catheter was placed for exchange transfusions. He responded well to exchange transfusion and was discharged from the hospital. $\mathrm{He}$ presented to his local physician at fifteen days of age with a mass in the right groin. He showed normal range of motion of both hips and was in no apparent discomfort. White blood cell count (WBC) was $8,700 / \mathrm{mm}^{3}$ and erythrocyte sedimentation rate (ESR) was $45 \mathrm{~mm} / \mathrm{h}$. Over the next $72 \mathrm{~h}$ the patient became febrile to $101.6^{\circ} \mathrm{F}$ and developed left knee swelling. Left knee radiograph revealed areas of bony destruction in the distal left femur consistent with osteomyclitis. Aspiration of the left knee yielded three milliliters of purulent fluid (cultures were positive for Staphylococcus aureus). $\mathrm{He}$ continued to have a right groin mass, but had full range of motion of the right hip. WBC and ESR rose to $20,000 / \mathrm{mm}^{3}$ and $53 \mathrm{~mm} / \mathrm{h}$, respectively, with blood cultures positive for Staphylococcus aureus. A CT of the pelvis revealed a retroperitoneal abscess on the right. At surgery, an ilio-psoas muscle abscess (which communicated with the right hip joint) was found. At the conclusion of six weeks of intravenous and oral antibiotic therapy, the WBC and ESR were normal. The patient has remained symptom-free for five years, with remodeling of the distal femur and normal ossification of the right femoral head. An evaluation for immunodeficiency was negative.

Correspondence to: Dr. A. E. Schlesinger

\section{Case 2}

This white male was born at 42 weeks by spontaneous vaginal delivery. The delivery and immediate postnatal course were unremarkable, and the infant was discharged from the hospital on the second day of life. At three weeks of age, his parents noted decreased movement of his right leg and a preference for holding it in a flexed position. Mild erythemia and swelling of the extremity developed over the next $24-48 \mathrm{~h}$. There was no fever or irritability. Physical examination revealed a slightly pale right leg with mild bluish discoloration. The leg was held flexed, and the patient showed signs of discomfort when the leg was moved. There was fullness of the right flank. On admission, the WBC and ESR were $18,600 / \mathrm{mm}^{3}$ and $1 \mathrm{~mm} / \mathrm{h}$, respectively. Blood cultures were negative. Sonography revealed an enlarged right ilio-psoas muscle. A pelvic CT scan revealed enlargement of the right ilio-psoas muscle with fluid attenuation centrally consistent with an abscess (Fig. 1). Over the next several hours, the patient's leg became increasingly edematous, discolored, and painful, with the WBC and ESR increasing to $26,000 / \mathrm{mm}^{3}$ and $31 \mathrm{~mm} / \mathrm{h}$, respectively. Aspiration of the ilio-psoas mass under $\mathrm{CT}$ guidance yielded 3-5 cc of purulent, thick, green fluid; cultures quickly grew Staphylococcus aureus. Surgical drainage was performed followed by a 14 day course of intravenous antibiotics. The child did well postoperatively. At time of discharge, WBC and ESR were normal, and there was full range of motion of the right lower extremity. He was continued on one week of oral antibiotics, and has been symptomfree for the past six months.

\section{Discussion}

Ilio-psoas abscess is rare in children, and exceedingly uncommon in the neonatal period. We know of only two prior reports of ilio-psoas abscess in the neonatal period $[1,2]$. Ilio-psoas abscess may occur primarily or can be secondary to inflammatory lesions of the spine or sacroiliac region, complications of surgery, gastrointestinal tract inflammation or perforation, extension of renal abscesses, or lymphatic spread of pelvic infections [1]. If the cultures grow Staphylococcus aureus, the abscess is presumed to be primary in origin, and multiple enteric organisms are usually cultured from secondary ilio-psoas abscesses [3]. Ilio-psoas abscesses are usually treated by surgical drainage, but percutaneous drainage has also been performed 

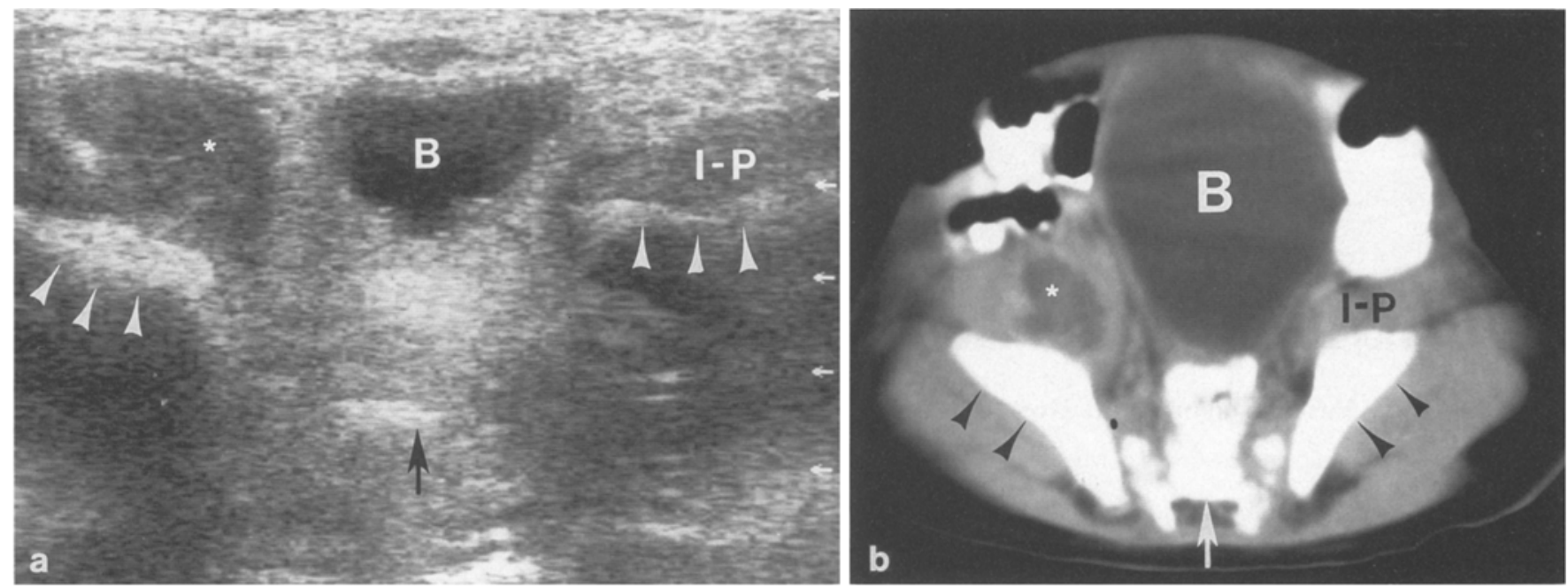

Fig.1. a Transverse (axial) image from a pelvic sonogram in case 2 reveals enlargement of the ilio-psoas muscle on the right $(*)$. The left ilio-psoas muscle is normal $(I-P)$. (B, bladder; arrowheads, iliac bone; arrow, sacrum). b Contast-enhanced axial CT scan in case 2 at

$[3,4]$. A recent report has advocated antibiotic therapy without drainage [5].

In the cases we describe, as well as the two previously reported neonatal cases, the patients had primary iliopsoas abscesses. Although no definite cause for such "spontaneous" primary psoas abscesses has been proven, postulated etiologies have included intramuscular hemorrhage due to the trauma of delivery or blood-borne infection such as in generalized sepsis [1]. It is interesting that one of our patients had an umbilical venous catheter in place in the perinatal period, raising the possibility of bacteremia as a cause of the ilio-psoas abscess. It is also curious that our patients and those previously reported all presented between two and three weeks of age. One might speculate that a psoas muscle hemorrhage at the time of birth or a bacteremic event in the immediate perinatal period may require two to three weeks to develop into a large enough ilio-psoas abscess to cause signs or symptoms sufficient to seek medical attention.

In summary, ilio-psoas abscess should be included in the differential diagnosis when an infant presents in the first the same level as the sonogram in Fig. 1 a confirms the ilio-psoas abscess on the right $(*)$. ( $B$, bladder; arrowheads, iliac bone; arrow, sacrum; $I-P$, left ilio-psoas muscle)

few weeks of life with decreased range of motion of the hip or with discoloration or swelling in the flank or hip. In such cases, CT or sonography can readily identify the abscess and can provide guidance for needle aspiration or for percutaneous drainage.

\section{References}

1. Schut JM, Meradji M, Oraje AP, Bergmeijer JH, Schuller JL (1988) Double-sided psoas abscess in a young infant: sonographic and radiographic findings. Pediatr Radiol 18: 176

2. Sedaghatian MR, Barkhordar J, Gerami S (1978) Retroperitoneal abscess presenting as an abdomenal mass in neonate. $J$ Pediatr Surg 13:544

3. Hoffer FA, Shamberger RC, Teele RL (1987) Ilio-psoas abscess: diagnosis and management. Pediatr Radiol 17: 23

4. Sullivan WT, Glasier CM, Yrizzary JM, Collins TL (1984) Percutaneous drainage of a psoas abscess in a young child. J Can Assoc Radiol 35: 324

5. Harway RA, Bravo RH, Main GD, Ward GR (1988) Psoas abscess successfully treated with antibiotics. Contemp Orthop 16: 95 